\title{
O uso de corantes alimentícios como tema gerador em aulas experimentais de Química
}

The use of food colorants as the generating theme in experimental Chemistry classes

\author{
C. R. De Almeida; G. De Oliveira; F. F. Da Silva; S. S. Muler; K. P. Nicolini; J. \\ Nicolini
}

Departamento de Química/LACOPPI - Laboratório de Corantes e Processos Pirolíticos, Instituto Federal do ParanáIFPR, CEP 85.555-000, Palmas-PR, Brasil

*jaqueline.nicolini@ifpr.edu.br

(Recebido em 23 de fevereiro de 2018; aceito em 15 de abril de 2018)

\begin{abstract}
Este artigo apresenta uma estratégia didático-pedagógica que utiliza as propriedades dos corantes alimentícios como tema gerador em aulas experimentais de Química para a introdução dos conceitos de espectroscopia de UV-Vis e cromatografia, visto que estas técnicas são complementares e apresentam-se hifenadas na cromatografia líquida, por exemplo. A partir de análises espectroscópicas e cromatográficas, foram determinados e comparados os parâmetros físico-químicos energia de transição molar $\left(\mathrm{E}_{\mathrm{T}}\right)$, absortividade molar ( $\varepsilon$ ) e os índices de retenção (Rf) dos corantes amaranto, amarelo de tartrazina e azul de indigotina. Observam-se pequenas alterações dos comprimentos de onda máximos dos corantes testados, com a variação da polaridade dos solventes utilizados, principalmente do corante azul de indigotina, o que reflete em diferentes valores de $\mathrm{E}_{\mathrm{T}}$. Com essa proposta de atividade experimental é possível fortalecer o processo de ensino e aprendizagem, de forma significativa, a qual está baseada na observação, na análise dos dados experimentais, seguida de uma conclusão, pautada pela contextualização entre o tema gerador e com o cotidiano dos estudantes.
\end{abstract}

Palavras-chave: corantes alimentícios, parâmetros físico químicos, processo ensino-aprendizagem, tema gerador

This manuscript shows a didactic-pedagogical throw food dyes theme in experimental Chemistry classes. From the results was possible to introduce some concepts about UV-Vis spectroscopy and chromatography. This is important because these two techniques complement each one, and are used hyphenated in liquid chromatography, for example. From spectroscopy and chromatography methods, the physicochemical parameters molar transition energy (ET), molar absorptivity $(\varepsilon)$ and retention indices (Rf) of amaranth, tartrazine yellow and indigo carmine dyes were determined and compared. Was observed little changes in the maximum wavelengths of the dyes tested, with the variation of the polarity of the solvents used, especially of the indigo carmine dye, bring forth different $\mathrm{E}_{\mathrm{T}}$ values obtained. With this proposal, it is possible to strengthen the teaching and learning process, significantly, based on observation, on the analysis of experimental data, and by a conclusion based on the contextualization with the theme and with daily life of the students.

Keywords: food dyes, physic-chemical parameters, teaching-learning process, generating theme

\section{INTRODUÇÃO}

Alguns corantes para fins alimentícios contém o grupo azo $(-\mathrm{N}=\mathrm{N}-)$, sendo potencialmente tóxicos ou carcinogênicos [1], provocando reações alérgicas [2], interagindo com a heme [3] e com albumina sérica humana [4]. Conforme descrito pela Organização de Agricultura e Alimentação Americana (Food and Agriculture Organization of the United Nations - FAO) o consumo de aditivos, na forma de corantes limita-se a alguns $\mathrm{mg} / \mathrm{kg}$ diários, dependendo da massa corpórea do indivíduo. A presença dos corantes como aditivos nos alimentos é indicada nos rótulos pela sigla INS que indica o número do corante de acordo como Sistema Internacional (International Numbering System - INS). Dentre os corantes alimentícios, destacamos o amarelo de tartrazina (INS 102) [5] com Ingestão Diária Aceitável (IDA) de $0-7,5 \mathrm{mg} / \mathrm{kg}$, o amaranto (INS 123) [6] e o azul de indigotina (INS 132) [7], ambos com IDA de $0-0,5 \mathrm{mg} / \mathrm{kg}$. O consumo excessivo de alimentos que contêm corantes pode causar transtornos de comportamento, hiperatividade em crianças $[8,9]$ e alergias às pessoas com sensibilidade aos componentes da fórmula [2].

$$
047101-1
$$


Nesta proposta de atividade experimental, nas atividades que envolviam o maior tempo de exposição aos solventes, foram utilizados solventes verdes os quais são definidos como aqueles que contribuem para o desempenho ambiental dos processos na indústria química, impactando em menores custos, maior segurança, menor risco à saúde e ao meio ambiente [10-14]. A abordagem didático-pedagógica utilizando como tema gerador corante alimentício permite correlacionar o tema espectroscopia e cromatografia com o cotidiano dos estudantes, tendo em vista que o consumo de alimentos coloridos é elevado e os corantes não tem valor nutricional, apenas visual. Dessa maneira, é importante discutir com os estudantes que a alimentação pode influenciar na saúde e qualidade de vida dos indivíduos [15-18], e em especial, destacar sobre o consumo desmedido de alimentos coloridos e suas consequências à saúde [8]. Isso porque o uso de temas geradores [19], a problematização [20] e a experimentação em química [21, 22], a partir de diferentes enfoques [2226] são fundamentais para a construção de conceitos que permitam aos estudantes compreender o mundo em que eles se encontram inseridos [20,21], apresenta caráter social, contextualizador e integrador instrumentalizando os estudantes envolvidos no processo a tornarem-se sujeitos ativos e reflexivos em sociedade, sendo essa uma ação facilitadora do processo de ensino e aprendizagem. Para Popper a prática docente parte da lógica, consistindo em repensar, sendo suas discussões pautadas basicamente na: 1) a hipótese; 2) nas condições iniciais; e 3) nas conclusões obtidas a partir da atividade realizada [27]. A busca por estratégias de ensino e aprendizagem que permitam aos estudantes correlacionar temas do seu cotidiano, que promovam a aprendizagem significativa dos conceitos químicos, com estratégias pautadas no método e rigor científico, contribuem para que o estudante desenvolva habilidade experimental, senso investigativo e possa tornar-se um cidadão capaz de interagir em seu contexto social de forma crítica e inovadora. As atividades experimentais demonstram a integração entre ensino e pesquisa, demonstrando a interdisciplinaridade entre os componentes curriculares através da contextualização [25].

Neste manuscrito foi utilizado o tema gerador "corantes alimentícios" em aulas experimentais de Química para a introdução dos conceitos de espectroscopia de UV-Vis e cromatografia, visto que estas técnicas são complementares e apresentam-se hifenadas na cromatografia líquida, por exemplo. Com essa atividade proposta foi possível obter e comparar os parâmetros físico-químicos energia de transição molar $\left(\mathrm{E}_{\mathrm{T}}\right)$, absortividade molar $(\varepsilon)$ e os índices de retenção (Rf) dos corantes amaranto, amarelo de tartrazina e azul de indigotina utilizando solventes ecologicamente recomendados, que de acordo com os princípios da Química Verde reduzem, eliminam ou não geram substâncias perigosas [28, 29], além de fortalecer o processo de ensino e aprendizagem, de forma significativa contextualizando o tema gerador com o cotidiano dos estudantes.

\section{MATERIAL E MÉTODOS}

A pesquisa realizada foi dedutiva investigativa [30] utilizando como estratégias de análise dos dados os métodos de cromatografia em papel e espectroscopia de UV-Vis, visto serem técnicas complementares e hifenadas. Além do teste estatístico ANOVA para os dados obtidos os valores de média e desvio padrão também foram calculados. Os objetos de investigação foram corantes alimentícios, utilizados como tema gerador para abordar os princípios conceituais de cromatografia e espectroscopia, fortalecendo assim o processo de ensino e aprendizagem, de forma significativa. A estratégia foi pautada na observação e na análise dos dados experimentais, seguida de uma conclusão e de uma contextualização entre o tema gerador e o cotidiano dos estudantes [27, 31]. Participaram desta investigação estudantes do Curso Técnico em Alimentos e do Curso de Licenciatura em Química. Foi utilizado como instrumento de coleta de dados a observação direta, a análise documental, a redação de relatórios de registo, a análise e a interpretação dos dados coletados e grupo focal [32].

Foram testados os corantes amaranto (AMR) (Sigma-Aldrich, pureza 85-95\%), amarelo de tartrazina (AMT) (Neon, pureza > $99 \%$ ) e azul de indigotina (AZI) (Sigma-Aldrich, pureza $85 \%$ ) individualmente. Os corantes alimentícios apresentam alta solubilidade em água e baixa solubilidade em etanol puro. Foram preparados, para cada corante alimentício testado, $5 \mathrm{~cm}^{3} \mathrm{de}$ solução aquosa estoque, na concentração de $1 \times 10^{-3} \mathrm{~mol} / \mathrm{L}$. Em seguida, foi retirada uma alíquota de $150 \mu \mathrm{L}$ e transferida para um balão volumétrico de $5 \mathrm{~cm}^{3}$ para o preparo das soluções na 
concentração de $3 \times 10^{-5} \mathrm{~mol} / \mathrm{L}$ em água destilada, metanol (MeOH) (Vetec, pureza 99,8 \%), etanol $(\mathrm{EtOH})(\mathrm{Neon}$, pureza 99,8 \%), $n$-propanol ( $n$ - $\mathrm{PrOH})$ (Neon, pureza 99,95\%), iso-propanol (iso$\mathrm{PrOH}$ ) (Neon, pureza 99,8 \%), acetona (ACE) (Neon, pureza 99,5\%), e na mistura binária entre água e etanol nas proporções de $50 \%$ (EtOH50) e $70 \%$ (EtOH70), para avaliar o comportamento dos corantes AMR, AMT e AZI nos solventes que geram menor impacto ambiental. Em seguida as soluções preparadas foram analisadas por espectroscopia de UV-Vis (PerkinElmer Lambda 365). Para os corantes alimentícios, amarelo (CAM) que contém amarelo de tartrazina, do corante azul (CAZ) que contém azul de indigotina e do corante bordô (CBO) que contém amaranto, preparouse separadamente $5 \mathrm{~cm}^{3}$ das soluções aquosas estoque, na concentração comum de $8 \mathrm{~g} / \mathrm{L}$. Os corantes alimentícios CAM, CAZ e CBO foram adquiridos em um mercado da região e por questões éticas, comerciais e legais não é apresentada a marca dos mesmos.

Para as análises por cromatografia em papel [33], foram cortadas tiras de papel qualitativo com porosidade de $14 \mu \mathrm{m}$, nas dimensões de $3 \mathrm{~cm}$ de largura, por $15 \mathrm{de} \mathrm{cm}$ de comprimento, sendo traçado um caminho de $10 \mathrm{~cm}$ usando-se um lápis. Um traço a $2 \mathrm{~cm}$ da base do papel indica o início da corrida cromatográfica e com o auxílio de uma pipeta de Pasteur foi adicionada uma gota, sem diluição, dos corantes alimentícios CAM, CAZ e CBO, em papéis distintos. Para a análise por cromatografia em papel dos corantes AMR, AMT e AZI (P.A.), utilizou-se uma gota da solução estoque $\left(1 \times 10^{-3} \mathrm{~mol} / \mathrm{L}\right)$, também com o auxílio de uma pipeta de Pasteur. A tira de papel foi presa ao vidro relógio com o auxílio de fita adesiva, utilizando-se $3 \mathrm{~cm}$ de papel qualitativo. As amostras foram analisadas separadamente, em triplicata, para a obtenção da média e do desvio padrão e dos Rf's de cada corante testado. Visando minimizar o impacto ambiental e não causar risco à saúde dos estudantes, baseados nos princípios da Química Verde, para a corrida cromatográfica foi utilizado quatro sistemas de solventes: água, etanol comercial nas graduações de $46{ }^{\circ} \mathrm{GL}, 70{ }^{\circ} \mathrm{GL}$ e $92,8^{\circ} \mathrm{GL}$. O que permite a reprodução do experimento em qualquer realidade escolar.

\section{RESULTADOS E DISCUSSÃO}

\subsection{Análise cromatográfica}

Nas análises por cromatografia em papel utilizou-se água como primeira escolha, por ser um solvente ecologicamente adequado [34]. O verdor químico de um experimento se caracteriza pelo baixo impacto ambiental, bem como pelos solventes que são utilizados [35]. Pensando no verdor químico desta estratégia, nas análises por cromatografia em papel, método de análise mais demorado e que iria expor o estudante por mais tempo aos solventes além do uso de água como eluente também foram utilizados etanol comercial nas graduações de 46,70 e $92,8^{\circ} \mathrm{GL}$ e em decorrência do comportamento dos corantes nestes solventes, foram calculados os parâmetros físico-químicos índices de retenção (Rf) e a polaridade relativa (PR) dos eluentes utilizados. Assim, foram comparados o comportamento dos corantes CAM, CAZ, CBO, AMT, AMR e AZI por cromatografia em papel (Tabela 1 e Figura 1).

É sabido que a separação cromatográfica, a partir da variação da polaridade do solvente, permite identificar uma miríade de compostos [23, 36-42]. A água possui índice de polaridade 9,0 e o etanol 5,2 [43]. No entanto, a mistura entre os solventes, faz com que as polaridades relativas (PR) sejam distintas [43], as quais podem ser determinadas pela Equação 1:

$$
P R=\frac{\left(\% \text { Sol }_{1} \times \text { P.Sol }_{1}\right)+\left(\% \text { Sol }_{.2} \times \text { P.Sol }_{2}\right)}{100}
$$

(Equação 1)

Sendo PR a polaridade relativa, \% Sol.1 a porcentagem do solvente 1, P.Sol.1 a polaridade do solvente 1, \% Sol.2 a porcentagem do solvente 2, P.Sol.2 a polaridade do solvente 2. Dessa maneira, tem-se que a polaridade relativa do etanol $46{ }^{\circ} \mathrm{GL}$ é 7,3 ; do etanol $70{ }^{\circ} \mathrm{GL}$ é 6,4 e do etanol 92,8 ${ }^{\circ} \mathrm{GL}$ é 5,5. Os valores dos índices de retenção (Rf's) obtidos a partir dos corantes alimentícios (P.A.) demonstram que o AMR e o AMT apresentam menor afinidade química pelo eluente com menor 
polaridade relativa (etanol $92,8{ }^{\circ} \mathrm{GL}$ ). Já o corante AZI P.A. apresenta menor amplitude nas alterações de Rf pela variação da polaridade relativa (Tabela 1).

Tabela 1: Comparação entre os índices de retenção dos corantes alimentícios (P.A.) e comerciais: AMR/CBO, AMT/CAM e AZI/CAZ

\begin{tabular}{ccccc}
\hline Corante & Água & Etanol $\mathbf{4 6}{ }^{\mathbf{O}} \mathbf{G L}$ & Etanol 70 & \\
\end{tabular}

Cor da mancha das amostras testadas: 1: Rosa. 2: Amarelo. 3: Azul. Há significância entre as amostras pelo teste ANOVA ( $\mathrm{p}<0,05 \%$ ). Os valores apresentados são os valores médios $(\bar{x})$ obtidos a partir da equação $\bar{x}=\frac{\left(\text { amostra }_{1}+\text { amostra }_{2}+\text { amostra }_{3}\right)}{3}$ e o número entre parênteses indica $\mathrm{o}$

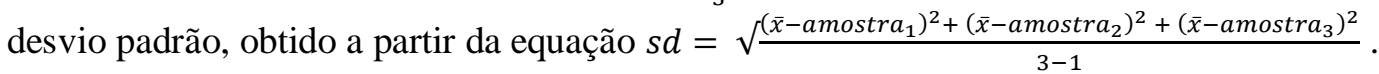

Todavia, quando se analisa o Rf dos corantes alimentícios comerciais que contêm AMR, AMT e AZI em sua constituição, observam-se Rf's distintos dos obtidos a partir dos corantes P.A., apresentados na Tabela 1. Todavia, há um padrão decrescente dos índices de retenção indicando que a afinidade química decresce com o decréscimo da polaridade. A variação da polaridade do solvente altera os índices de retenção obtidos bem como a velocidade de eluição, parâmetros importantes em uma análise cromatográfica [42]. A variação dos constituintes das amostras (corantes comerciais e P.A) faz com que o índice de retenção (Rf) seja distinto, essa flutuação nos valores de $\mathrm{Rf}$ pode ser explicada pela constituição dos corantes alimentícios comerciais que contêm água e álcool etílico além dos corantes AMR, AMT ou AZI. A proporção destes solventes não é indicada no rótulo dos corantes alimentícios utilizados. Isso influencia nas propriedades físicas índice de refração, densidade e parâmetros termodinâmicos; nas forças de interação intermoleculares; na solvatação seletiva ou micelar; e nos processos de ionização ou dissociação $[44,45]$, alterando os valores dos índices de retenção (Rf's) obtidos.

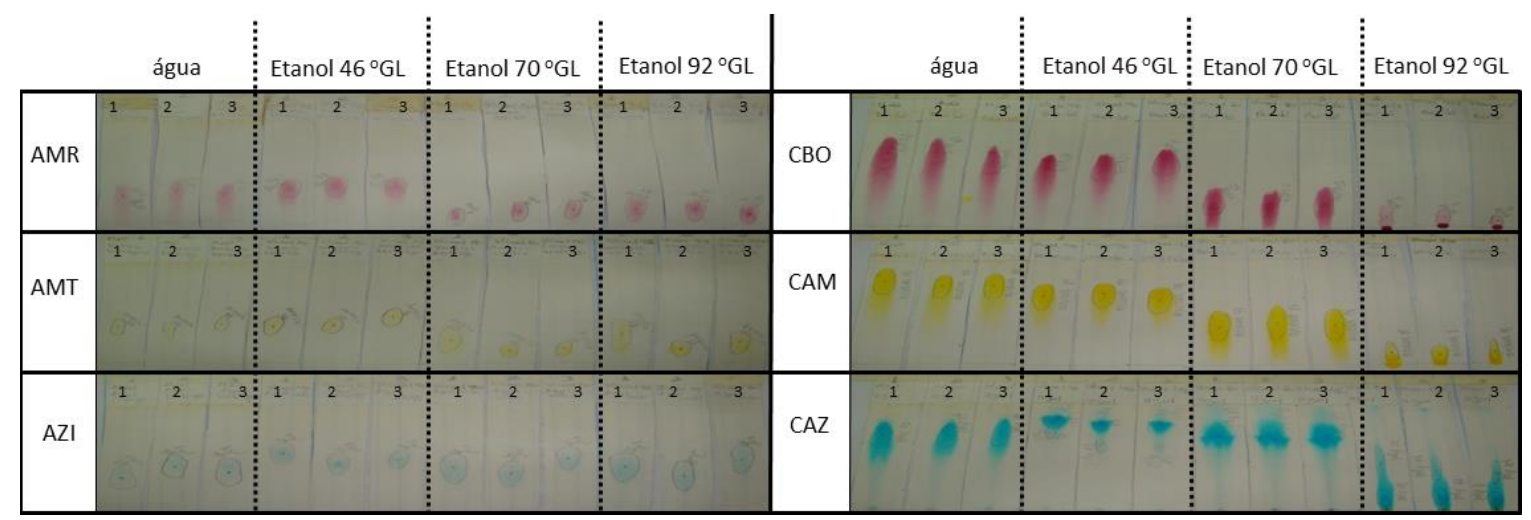

Figura 1: Perfil cromatográfico típico obtido a partir dos corantes P.A. amaranto (AMR), amarelo de tartrazina (AMT) e azul de indigotina (AZI) e dos corantes comerciais bordo (CBO), amarelo (CAM) e azul (CAZ), utilizando como eluente água, etanol $46^{\circ} \mathrm{GL}$, etanol $70^{\circ} \mathrm{GL}$ e etanol $92,8^{\circ} \mathrm{GL}$

Essa estratégia apresenta-se como uma ferramenta capaz de ressignificar $[19,46]$ a química e suas competências, desenvolvendo assim as habilidades necessárias para o estudante sentir-se parte do processo e instrumentalizado a agir em sociedade apropriando-se dos conceitos inerentes à química.

\subsection{Análise por espectroscopia de UV-Vis}


Através dos resultados obtidos por espectroscopia de UV-Vis foram comparados os parâmetros físico químicos de absortividadade molar $(\varepsilon)$ e a energia de transição molar $\left(\mathrm{E}_{\mathrm{T}}\right)$ dos corantes AMT, AMR e AZI (Tabela 2). Utilizou-se como solventes água, etanol, a mistura binária entre etanol e água nas proporções de 50 e $70 \%$, além de metanol, $n$-propanol, iso-propanol e acetona, com vistas a comparar as diferenças espectrais de cada corante em cada solvente testado.

A partir da Equação 2, é possível calcular a absortividade molar $(\varepsilon)$, a qual é definida pela capacidade de um mol de substância em absorver luz a um dado comprimento de onda [47]. A partir da Equação 3, é possível observar que a energia de transição molar $\left(\mathrm{E}_{\mathrm{T}}\right)$ e o comprimento de onda máximo $\left(\lambda_{\max }\right)$ são propriedades inversamente proporcionais, o que justifica os menores valores de $\mathrm{E}_{\mathrm{T}}$ com o aumento do $\lambda_{\max }$ observados na Tabela 2. Rocha \& Teixeira (2004) [48] apresentam exemplos de compostos que apresentam esse comportamento. Observa-se que dentre os corantes investigados o corante AZI, apresenta as maiores variações de $\mathrm{E}_{\mathrm{T}}$, tendo os maiores deslocamentos do $\lambda_{\max }$ com a variação da polaridade dos solventes, alterando fracamente a energia de transição molar $\left(\mathrm{E}_{\mathrm{T}}\right)$ (Equação 3).

Tabela 2: Energia de transição molar $\left(E_{T}\right)$ e absortividade molar $(\varepsilon)$ calculadas para os corantes P.A. em diferentes solventes

\begin{tabular}{ccccccccccc}
\hline \multirow{2}{*}{ Solvente } & \multirow{2}{*}{$\mathbf{P R}$} & \multicolumn{4}{c}{ AZI } & \multicolumn{3}{c}{ AMR } & \multicolumn{3}{c}{ AMT } \\
\cline { 3 - 11 } & & $\boldsymbol{\lambda}_{\max }$ & $\mathbf{E}_{\mathbf{T}}$ & $\boldsymbol{\varepsilon}$ & $\boldsymbol{\lambda}_{\max }$ & $\mathbf{E}_{\mathbf{T}}$ & $\boldsymbol{\varepsilon}$ & $\boldsymbol{\lambda}_{\max }$ & $\mathbf{E}_{\mathbf{T}}$ & $\boldsymbol{\varepsilon}$ \\
\hline Água & 9,0 & 611,5 & 46,76 & 29610 & 521,2 & 54,86 & 20203 & 428,9 & 66,66 & 8017 \\
EtOH50 & 7,2 & 614,6 & 46,52 & 31457 & 522,8 & 54,69 & 22367 & 430,9 & 66,35 & 8007 \\
MeOH & 6,7 & 600,3 & 47,63 & 31717 & 521,2 & 54,86 & 21557 & 428,2 & 66,77 & 7700 \\
EtOH70 & 6,4 & 612,9 & 46,65 & 31473 & 522,0 & 54,77 & 21837 & 430,9 & 66,35 & 8100 \\
ACE & 5,5 & 601,2 & 47,56 & 13823 & 523,1 & 54,66 & 15243 & 413,5 & 69,14 & 4383 \\
EtOH & 5,3 & 604,2 & 47,32 & 30917 & 523,7 & 54,59 & 21463 & 430,9 & 66,35 & 7903 \\
iso-PrOH & 4,4 & 603,1 & 47,41 & 15457 & 522,3 & 54,74 & 16567 & 423,7 & 67,48 & 6090 \\
$n$-PrOH & 4,2 & 606,7 & 47,13 & 16133 & 526,2 & 54,33 & 23173 & 429,9 & 66,51 & 7980 \\
\hline
\end{tabular}

PR: Polaridade relativa da solução, calculada a partir da Equação 1. $\varepsilon$ : em L/mol cm, calculada a partir da Equação 2. $\mathrm{E}_{\mathrm{T}}$ : em kcal/mol, calculada a partir da Equação 3. Há significância entre as amostras pelo teste ANOVA ( $\mathrm{p}<0,05 \%)$. Os valores apresentados são os valores médios $(\bar{x})$

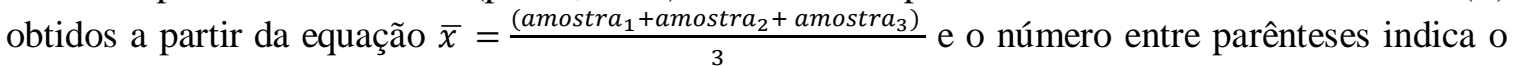

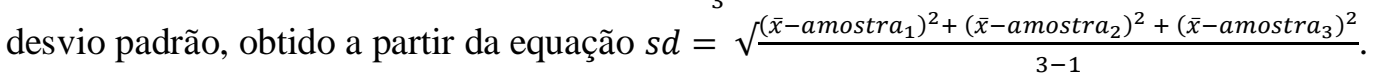

$$
\varepsilon=\frac{A}{b c}
$$

(Equação 2)

$$
E_{T}=\frac{28591}{\lambda_{\max }(\mathrm{nm})}
$$

(Equação 3)

A espectroscopia de UV-Vis limita-se a moléculas que apresentam sistemas conjugados [47], mas que pode ser aplicado a várias situações problema, desde o monitoramento de parâmetros ambientais [49], questões relacionadas à saúde [50,51] ou na alteração e estudo de novos materiais $[52,53]$. As moléculas que apresentam sistemas conjugados ou insaturados estão presentes em sistemas químicos, físicos e/ou biológicos em amostras sólidas, líquidas ou gasosas [54-59]. Os espectros de ultravioleta correlacionam a intensidade de absorção com o comprimento de onda máximo da amostra. A partir da investigação descrita neste manuscrito, observa-se que a proporção 
entre água:etanol na mistura binária, influencia na posição da banda de absorção do corante AZI, com os valores de $\lambda_{\max }$ em ordem crescente EtOH $<\mathrm{H}_{2} \mathrm{O}<$ EtOH70 $<$ EtOH50. A energia de transição molar $\left(\mathrm{E}_{\mathrm{T}}\right)$ pode ser calculada apenas para os corantes P.A., pois os corantes comerciais apresentam concentração dos corantes testados desconhecida.

Com a aquisição dos espectros, neste caso é possível discutir que cada cor implica em um diferente comprimento de onda, permitindo ao estudante associar a técnica com a extração de pimentos vegetais e seus constituintes [22], por exemplo, e assim correlacionar com a cromatografia em papel [23], compreendendo a importância e a possibilidade de correlação que as duas técnicas apresentam.

Dessa maneira, evidencia-se que o uso de temas geradores em aulas experimentais de Química permitem que o estudante desenvolva habilidades práticas, de raciocínio e de lógica científica, pautadas pelo método e pelo rigor científico [27, 60, 61]. Assim, o uso de um tema gerador contribui para despertar a curiosidade do estudante, desafiando-o a observar a realidade, entendê-la e modificá-la [20,46]. Além disso, durante a redação de relatórios de registo, análise e interpretação dos dados coletados os estudantes apresentaram afirmações e questionamentos, tais como:

Estudante 1: "Posso fazer isso com qualquer corante?"

Estudante 2: "Estou achando essa atividade bem legal, pois como futura professora eu posso aplicar essa atividade, principalmente a de cromatografia, em escolas da rede pública com diferentes realidades."

Estudante 3: "Estou gostando muito de fazer esse experimento! Eu não pensava que era possível fazer isso."

Estudante 4: "Das atividades que fiz até agora, essa é a que eu mais gostei professora."

A partir dessa atividade experimental, o professor pode abordar vários tópicos, dependendo da disciplina e do enfoque, tais como: Polaridade, polaridade relativa, eluente, fase móvel, fase estacionária, cor, energia, comprimento de onda, absortividade molar, energia de transição molar, aprendizagem significativa, qualidade de vida, alimentação e saúde.

Com essa proposta de atividade experimental é possível fortalecer o processo de ensino e aprendizagem, de forma significativa, a qual está baseada na observação, na análise dos dados experimentais, seguida de uma conclusão, pautada pela contextualização entre o tema gerador e com o cotidiano dos estudantes.

\section{CONCLUSÃo}

Este artigo apresenta uma investigação acerca do uso de corantes alimentícios como tema gerador para a introdução dos conceitos relacionados às técnicas de espectroscopia e cromatografia. A partir da abordagem geradora proposta nessa atividade experimental pode-se instrumentalizar os estudantes a resolver problemas cotidianos. Ainda podem-se propor questões para discussão com os estudantes abordando: a) o que foi observado variando o eluente em uma análise cromatográfica e espectroscópica; b) os conceitos fundamentais das técnicas de cromatografia: eluente, eluato, índice de retenção, velocidade de eluição, separação de misturas; c) os princípios fundamentais da espectroscopia de UV- Vis entre a posição da banda de absorção, a cor da amostra e a energia de transição molar; a intensidade da banda de absorção, a concentração da amostra e a absortividade molar. Estabelecendo assim correlações matemáticas entre um fenômeno observado e a aplicação das equações propostas; d) a elaboração de um texto, baseado nas experiências cotidianas dos estudantes que envolvam corantes e a separação de compostos e assim e) o aumento do espaço de discussão a partir das experiências vividas pelos estudantes.

A partir dos dados obtidos foi comparado e abordado o comportamento físico-químico dos corantes alimentícios amaranto, azul de indigotina e amarelo de tartrazina procurando minimizar o impacto ao meio ambiente e para a saúde, usando-se água na maior parte das atividades, conforme recomendado pelos princípios da Química Verde. Os resultados obtidos também demonstram que variando a polaridade do solvente há variação do índice de retenção (Rf) de cada amostra e variação da energia de transição molar $\left(\mathrm{E}_{\mathrm{T}}\right)$ e consequentemente na absortividade molar $(\varepsilon)$. Este artigo apresenta-se como uma ferramenta didático-pedagógica para abordar os conceitos fundamentais de cromatografia e de espectroscopia de UV-Vis em aulas experimentais de Química Geral em cursos 
de Química e ainda em cursos de Biologia e Farmácia ou em Cursos Técnicos que contenham química em sua matriz curricular.

\section{AGRADECIMENTOS}

Ao CNPq, ao IFPR, ao Programa Institucional de Apoio à Pesquisa (PIAP/IFPR - Edital 023/2014), ao PIBIC-Jr/CNPq e PIBIC-Af/CNPq.

\section{REFERÊNCIAS BIBLIOGRÁFICAS}

1. Chung KT. Mutagenicity and carcinogenicity of aromatic amines metabolically produced from azo dyes. J. Environ. Sci. Health, Part C: Environ Carcinog Rev. 2000;18(1):51-74, doi: $10.1080 / 10590500009373515$.

2. Agência Nacional de Vigilância Sanitária (ANVISA). Resolução Anvisa N. 30. Informe Técnico no .30 , de 24 de julho de 2007 - Considerações sobre o corante amarelo tartrazina. Disponível em: http://portal.anvisa.gov.br/documents/33916/388729/Informe+T\%C3\%A9cnico+n\%C2\%BA+30\%2C+ de+24+de+julho+de+2007/d47a1 fea-fd03-4e94-8dff-fd87d3b1296a. Acesso em 21 de fevereiro de 2018.

3. Basu A, Kumar GS. Interaction of toxic azo dyes with heme protein: Biophysical insights into the binding aspect of the food additive amaranth with human hemoglobin. J Hazard Mater. 2015;289(-):204-209, doi: http://doi.org/10.1016/j.jhazmat.2015.02.044.

4. Zhang G, Ma Y. Mechanistic and conformational studies on the interaction of food dye amaranth with human serum albumin by multispectroscopic methods. Food Chem. 2013;136(2):442-449, doi:http://doi.org/10.1016/j.foodchem.2012.09.026.

5. Food and Agriculture Organization of United Nations (FAO). Amarelo de tartrazina. Disponível em: http://www.fao.org/ag/agn/jecfa-additives/specs/Monograph1/Additive-458.pdf. Acesso em: $21 \mathrm{de}$ fevereiro de 2018.

6. Food and Agriculture Organization of United Nations (FAO). Amaranto. Disponível em: http://www.fao.org/ag/agn/jecfa-additives/specs/Monograph1/Additive-018.pdf. Acesso em: $21 \mathrm{de}$ fevereiro de 2018.

7. Food and Agriculture Organization of United Nations (FAO). Indigontina. Disponível em: http://www.fao.org/ag/agn/jecfa-additives/specs/Monograph1/Additive-234.pdf. Acesso em: 21 de fevereiro de 2018.

8. Sá P, Ferreira FA, Nova RDV, Mourão TV, Andrade VLÂ, Rückl S. Uso abusivo de aditivos alimentares e transtornos de comportamento: há uma relação? Int J Nutrology. 2016;9(2):209-215.

9. McCann D, Barrett A, Cooper A, Crumpler D, Dalen L, Grimshaw K, Kitchin E, Lok K, Porteous L, Prince E, Sonuga-Barke E, Warner JO, Stevenson J. Food additives and hyperactive behaviour in 3-yearold and 8/9-year-old children in the community: a randomised, double-blinded, placebo-controlled trial. The Lancet. 2007;370(9598):1560-1567, doi:http://doi.org/10.1016/S0140-6736(07)61306-3.

10. Capello C, Fischer U, Hungerbühler K. What is a green solvent? A comprehensive framework for the environmental assessment of solvents. Green Chem. 2007;9(9):927-934, doi:10.1039/b617536h.

11. Byrne FP, Jin S, Paggiola G, Petchey THM, Clark JH, Farmer TJ, Hunt AJ, Robert McElroy C, Sherwood J. Tools and techniques for solvent selection: green solvent selection guides. Sustainable Chem Processes. 2016;4(1):7, doi:10.1186/s40508-016-0051-z.

12. Buka I, Osornio-Vargas A, Clark B. Food additives, essential nutrients and neurodevelopmental behavioural disorders in children: A brief review. Paediatr Child Healt. 2011;16(7):54-56, doi:10.1093/pch/16.7.e54.

13. Bosiljkov T, Dujmić F, Cvjetko Bubalo M, Hribar J, Vidrih R, Brnčić M, Zlatic E, Radojčíc Redovniković I, Jokić S. Natural deep eutectic solvents and ultrasound-assisted extraction: Green approaches for extraction of wine lees anthocyanins. Food Bioprod Process. 2017;102(-):195-203, doi:http://doi.org/10.1016/j.fbp.2016.12.005.

14. Ingold M, Dapueto R, Lopez GV, Porcal W. Una reacción multicomponente verde en el laboratorio de química orgánica. Educ Quim. 2016;27(1):15-20, doi:10.1016/j.eq.2015.09.008.

15. Spittle B. Neurotoxic effects of fluoride. Int Clin Psychopharm. 2004;44(3):117-124.

16. Raskin I, Ripoll C. Can an apple a day keep the doctor away? Curr Pharm Des. 2004;10(27):3419-3429.

17. Exley C, Vickers T. Elevated brain aluminium and early onset Alzheimer's disease in an individual occupationally exposed to aluminium: a case report. J Med Case Rep. 2014;8(1):1. 
18. Bernardi F, Nicolini KP, Nicolini J. Use of UV-Vis spectroscopy to detection of quercetin in plants by formation of quercetin-aluminum complex. Infarma. 2017;29(3):271-276, doi:http://dx.doi.org/10.14450/2318-9312.v29.e1.a2017.pp68-80.

19. Miranda ACG, Pazinato MS, Braibante MEF. Temas geradores através de uma abordagem temática Freireana: Contribuições para o Ensino de Ciências. Rev Ed Cie Mat. 2017;7(3):73-92.

20. de Andrade RA, de Medeiros Simões AS. Drogas: uma proposta de metodologia da problematização no Ensino de Química. Rev Thema. 2018;15(1):5-24, doi:http://dx.doi.org/10.15536/thema.15.2018.524.573.

21. Martins RdC, Bernardi F, Dangui Kreve Y, Nicolini KP, Nicolini J. Coleção de propostas utilizando produtos naturais para a introdução ao tema ácido-base no Ensino Médio (Parte I). Educ Quim. 2017;28():246-253, doi:https://doi.org/10.1016/j.eq.2017.03.005.

22. Bernardi F, Nicolini KP, Nicolini J. Estudo fitoquímico de Hydrangea sp. por meio de métodos clássicos de análise por espectroscopia no ultravioleta visível (UV-Vis) e cromatografia em coluna e em papel. Infarma. 2017;29(1):68-70, doi:http://dx.doi.org/10.14450/2318-9312.v29.e1.a2017.pp68-80.

23. Ribeiro NM, Nunes CR. Análise de pigmentos de pimentões por cromatografia em papel. Quim Nova Esc. 2008;29(ago):30-37.

24. Oliveira RJ. Ensino de Química: Por Um Enfoque Epistemológico e Argumentativo. Quim Nova Esc. 2015;37(4):257-263, doi:http://dx.doi.org/10.5935/0104-8899.20150047.

25. Lustosa MAFS. Compostagem como proposta didática para falar sobre solos no ensino fundamental. Scientia Plena. 2017;13(12):1-9, doi:10.14808/sci.plena.2017.121701.

26. Lima ACdS, Afonso JC. A química do refrigerante. Quim Nova Esc. 2009;31(3):210-215.

27. Popper KR. A lógica da pesquisa científica. Cultrix: São Paulo; 1985.

28. Green Chemistry. Disponível em: www.epa.gov/greenchemistry. Acesso em: 16 set 2017.

29. Lenardão EJ, Freitag RA, Dabdoub MJ, Batista ACF, Silveira CdC. "Green chemistry": os 12 princípios da química verde e sua inserção nas atividades de ensino e pesquisa. Quim Nova. 2003;26(-):123-129, doi:10.1590/S0100-40422003000100020.

30. Marconi MDA, Lakatos EM. Fundamentos de metodologia científica. 8nd ed., Atlas: São Paulo; 2017.

31. da Silveira FL. A filosofia de Karl Popper e suas implicações no ensino da ciência. A filosofia de Cad Cat Ens Fís. 1989;6(2):148-162.

32. Barbosa EF. Instrumentos de coleta de dados em pesquisas educacionais. Educativa. 1998:out.:1-5.

33. Okumura F, Soares MHFB, Cavalheiro ÉTG. Identificação de pigmentos naturais de espécies vegetais utilizando-se cromatografia em papel. Quim Nova. 2002;25(4):680-683, doi:10.1590/S010040422002000400025.

34. Hartonen K, Riekkola M-L. The Application of Green Solvents in Separation Processes. 1nd ed., Elsevier; 2017.

35. Gonçalves FP, Yunes SF, Guaita RI, Marques CA, Pires TCM, Pinto JRM, Machado AASC. La dimensión ambiental de la experimentación en la enseñanza de la química: consideraciones sobre el uso de la métrica holística «estrella verde». Educ Quim. 2017;28(2):99-106, doi:http://dx.doi.org/10.1016/j.eq.2016.11.005.

36. Collins CH, Braga GL, Bonato PS. Fundamentos de Cromatografia. 1nd ed., Campinas: Editora da Unicamp; 2006.

37. Gosetti F, Gianotti V, Angioi S, Polati S, Marengo E, Gennaro M. Oxidative degradation of food dye E133 Brilliant Blue FCF: liquid chromatography-electrospray mass spectrometry identification of the degradation pathway. J Chromatogr A. 2004;1054(1):379-387, doi:10.1016/j.chroma.2004.07.106.

38. Gosetti F, Gianotti V, Polati S, Gennaro MC. HPLC-MS degradation study of E110 Sunset Yellow FCF in a commercial beverage. J. Chromatogr. A, 2005; 1090(-):107-115,doi: 10.1016/j.chroma.2005.07.024.

39. Minioti KS, Sakellariou CF, Thomaidis NS. Determination of 13 synthetic food colorants in water-soluble foods by reversed-phase high-performance liquid chromatography coupled with diode-array detector. Anal Chim Acta. 2007;583(1):103-110, doi:10.1016/j.aca.2006.10.002.

40. Fabbri D, Adamiano A, Torri C. GC-MS determination of polycyclic aromatic hydrocarbons evolved from pyrolysis of biomass. Anal Bioanal Chem. 2010;397(1):309-317, doi:10.1007/s00216-010-3563-5.

41. Pugajeva I, Rozentale I, Viksna A, Bartkiene E, Bartkevics V. The application of headspace gas chromatography coupled to tandem quadrupole mass spectrometry for the analysis of furan in baby food samples. Food Chem. 2016;212(20-26), doi:10.1016/j.foodchem.2016.05.159.

42. Degani ALG, Cass QB, Vieira PC. Cromatografia um breve ensaio. Quim Nova Esc. 1998;7:21-25.

43. Schirmer RE. Modern Methods of Pharmaceutical Analysis. 2a ed., CRC Press; 1982.

44. Katritzky AR, Fara DC, Yang H, Tämm K, Tamm T, Karelson M. Quantitative measures of solvent polarity. Chem Rev. 2004;104(1):175-198, doi:10.1021/cr020750m.

45. Russel JB. Química Geral - Volume 1. 2nd ed., São Paulo: Pearson Makon Books; 1994.

46. Bachelard G. A Epistemologia. Edições 70: Lisboa; 2000. 
47. Silverstein RM, Webster FX. Identificação espectrométrica de compostos orgânicos. Rio de Janeiro: Guanabara Koogan; 2000.

48. Rocha FRP, Teixeira LSG. Estratégias para aumento de sensibilidade em espectrofotometria UV-VIS. Quim Nova. 2004;27(5):807-812, doi:10.1590/S0100-40422004000500021.

49. Figueiró CSM, Bastos de Oliveira D, Russo MR, Caires ARL, Rojas SS. Fish farming water quality monitored by optical analysis: The potential application of UV-Vis absorption and fluorescence spectroscopy. Aquaculture. 2018;490(91-97), doi:https://doi.org/10.1016/j.aquaculture.2018.02.027.

50. Saha M, Nandy P, Chakraborty M, Das P, Das S. The importance of pKa in an analysis of the interaction of compounds with DNA. Biophys Chem. 2018;236:15-21, doi:https://doi.org/10.1016/j.bpc.2018.02.001.

51. Coronetti MR, Cortes ALM, Franzen M, Hosoi BMU, Alves GN, Nicolini J, Nicolini KP. Avaliação in vitro de alterações em Lactuca spp. de Saccharomyces cereviseae na presença de fármacos. Infarma. 2017;29(2):133-140, doi:http://dx.doi.org/10.14450/2318-9312.v29.e2.a2017.pp133-140.

52. Zanquetti A, Finkler AC, Hosoi BMU, Nicolini J, Nicolini KP. Influência do tratamento hidrotérmico na energia de band gap de carvões de Eucaliptus spp. Rev Thema. 2018;15(1):25-33, doi:http://dx.doi.org/10.15536/thema.15.2018.25-33.761.

53. Guimarães AR, Cordeiro ME, Nicolini J, Nicolini KP. Use of ultrasound to modify the pyrolyzed biomass of Pinus spp. and the implications for biological models. Inf Process Agr. 2018;5(2):199-204, https://doi.org/10.1016/j.inpa.2018.03.001.

54. Bate-Smith E, Davenport SM, Harborne J. Comparative biochemistry of flavonoids-III.: A correlation between chemistry and plant geography in the genus Eucryphia. Phytochemistry, 1967; 6(10):14071413,doi: 10.1016/S0031-9422(00)82883-6.

55. Lichtenthaler HK. Chlorophylls and carotenoids: Pigments of photosynthetic biomembranes. Methods Enzymol. 1987;148(34):350-382, doi:10.1016/0076-6879(87)48036-1.

56. Linn MM, Poncio DC, Machado VG. An anionic chromogenic sensor based on the competition between the anion and a merocyanine solvatochromic dye for calix[4]pyrrole as a receptor site. Tetrahedron Lett. 2007;48(26):4547-4551, doi:https://doi.org/10.1016/j.tetlet.2007.04.141.

57. Mercê ALR, Nicolini J, Khan MA, Bouet G. Qualitative study of supramolecular assemblies of bcyclodextrin and cholecalciferol and the cobalt (II), copper (II) and zinc (II) ions. Carbohydr Polym. 2009;77(-):402-409, doi:10.1016/j.carbpol.2009.01.019.

58. Bi H, Duarte CM, Brito M, Vilas-Boas V, Cardoso S, Freitas P. Performance enhanced UV/vis spectroscopic microfluidic sensor for ascorbic acid quantification in human blood. Biosens Bioelectron. 2016;85(-):568-572, doi:http://dx.doi.org/10.1016/j.bios.2016.05.054.

59. Edinger P, Schneebeli J, Struis RPWJ, Biollaz SMA, Ludwig C. On-line liquid quench sampling and UVVis spectroscopy for tar measurements in wood gasification process gases. Fuel. 2016;184(-):59-68, doi:http://dx.doi.org/10.1016/j.fuel.2016.06.127.

60. de Souza PVT, Silva MD, Amauro NQ, Mori RC, Moreira PFdSD. Densidade: Uma Proposta de Aula Investigativa. Quim Nova Esc. 2015;37(2):120-124, doi:http://dx.doi.org/10.5935/0104-8899.20150028.

61. Barberà $\mathrm{O}$, Valdés $\mathrm{P}$. El trabajo práctico en la enseñanza de las ciencias: una revisión. El trabajo práctico en la enseñanza de las ciencias: una revisión. 1996;14(3):365-379. 